\title{
Protée
}

\section{La patine et la connivence}

\section{Jacques Fontanille}

Volume 29, numéro 1, 2001

La société des objets. Problèmes d'interobjectivité

URI : https://id.erudit.org/iderudit/030613ar

DOI : https://doi.org/10.7202/030613ar

Aller au sommaire du numéro

\section{Éditeur(s)}

Département des arts et lettres - Université du Québec à Chicoutimi

\section{ISSN}

0300-3523 (imprimé)

1708-2307 (numérique)

Découvrir la revue

\section{Citer cet article}

Fontanille, J. (2001). La patine et la connivence. Protée, 29(1), 23-35. https://doi.org/10.7202/030613ar

\section{Résumé de l'article}

La patine est tout d'abord affaire de temps, et en cela elle procure à l'objet une surface (sémiotique) d'inscription pour les « empreintes » d'un ensemble d'usages (les énonciations antérieures et successives des objets). Elle participe donc d'une mémoire figurative des objets, car, tout comme le discours verbal le fait avec la langue, l'énonciation des objets les modifie, et inscrit ces modifications dans leur structure même, qui en garde mémoire. En outre, cette mémoire, en ancrant les objets dans une sorte de tradition matérielle, celle de la longue chaîne des énonciations successives, projette des systèmes de valeur qui motivent leur co-existence : la mémoire inscrite dans la patine de chacun d'eux, devient alors leur mémoire collective, première étape de l'interobjectivité. Mais, pour pouvoir parler d'“ interobjectivité », il faut d'abord pouvoir parler d' " objets ", c'est-à-dire d'" actants ». Et on s'aperçoit alors que la patine, en dotant les « choses " soumises aux temps et aux usages d'une " enveloppe " chargée de valeurs et de mémoire, les convertit d'abord en actants compétents. Cette " compétence " n’est pas seulement de type sémantique (les valeurs incarnées dans la matière par les usages), elle est aussi modale, en ce sens que la patine et l'usure, tout comme l'ergonomie, induisent des contraintes et des lignes de tendance, des formes qui modalisent l'usager et infléchissent l'usage.
Ce document est protégé par la loi sur le droit d'auteur. L'utilisation des services d'Érudit (y compris la reproduction) est assujettie à sa politique d'utilisation que vous pouvez consulter en ligne.

https://apropos.erudit.org/fr/usagers/politique-dutilisation/ 


\section{LA PATIN E ET LA CO N N IVEN CE}
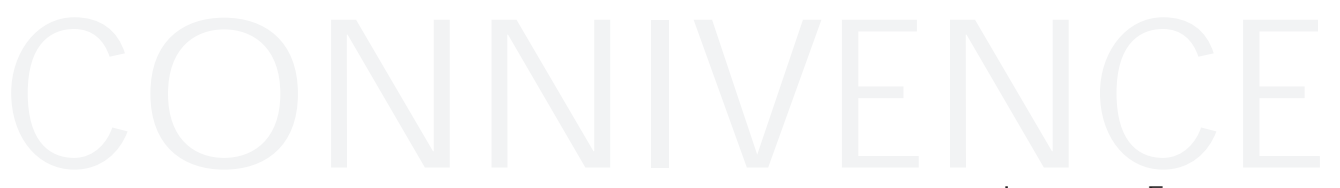

JACQUES FONTANILLE

La patine est, au sens propre, une couche de composé de cuivre qui se forme avec le temps sur les objets qui contiennent une part de ce métal. Par extension, le mot désigne toute altération superficielle et régulière que le temps impose aux objets constitués d'une matière dure, stable et en général immuable.

En tant que phénomène, la patine est donc à la fois une expression du «temps qui passe» et de l'usage, inscrits sur la surface extérieure des objets, et l'expression du "temps qui dure», ce dont témoignent la solidité et la permanence de la matière et de la structure intérieures des objets. «À la fois» et nécessairement, car le premier présuppose le second: la perception des effets du "temps qui passe» comme patine repose sur un postulat de permanence et de consistance intérieure de l'objet, sans quoi il n'y aurait plus «patine», mais, tout simplement, usure ou destruction.

Par ailleurs, si on imagine une collection d'objets ayant cohabité dans un même lieu, subi les mêmes usages, fréquenté les mêmes usagers et rencontré les mêmes passants, connu les mêmes aléas climatiques et sociaux, et ayant également résisté à tout cela, alors la patine inscrite sur leur enveloppe extérieure est non seulement la trace de tous ces événements multiples et répétés, de leur passé de cohabitation, mais aussi la manifestation (1) d'une coexistence continue, d'une longue quotidienneté partagée, et saisie en quelque sorte d'un seul regard ou d'un seul contact, (2) d'une mémoire collective des objets, inscrite dans leur matière même, qui marque ainsi la constitution d'une «interobjectivité», élaborée dans le temps et/ou par l'usage, et dans l'interaction avec l'ensemble des usagers ${ }^{1}$.

Mais, par ailleurs, ce qui constitue la "communauté» d'un ensemble d'objets, c'est aussi celle, synchronique et diachronique, de leurs usagers. Cette "enveloppe patinée» qui les rend similaires et familiers découle indirectement d'une relation avec les sujets humains, usagers, voisins ou passants, relation qui, en quelque sorte, les «humanise».

De fait, pour pouvoir parler d' «interobjectivité», il faut d'abord pouvoir parler d'«objets», c'est-à-dire d' «actants». Cette réflexion sur l'interobjectivité doit donc être replacée dans la perspective de l' "actantialisation» des choses qui peuplent notre monde: comment nous y prenons-nous pour faire des «objets» avec des 
"choses"? Les choses sont des corps matériels, qui partagent cette propriété avec le corps même des sujets, mais toute la question est de savoir comment ils peuvent partager plus encore avec eux: comment, plus précisément, peuvent-ils être saisis comme des "corps-actants", au même titre que le corps des sujets. Pour répondre à cette dernière question, il faudra s'interroger sur le statut de cette "enveloppe» de patine, véritable surface d'inscription sémiotique, rétensive et protensive, qui témoigne des usages antérieurs, qui a reçu l'empreinte du corps des usagers, et qui, de ce fait même, invite à de nouveaux usages et dessine la place et la forme d'autres contacts.

\section{EFFETS TEMPORELS: EMPREINTE ET ÉNONCIATION}

L'enveloppe et la surface d'inscription

Certes, conformément à sa définition rappelée en introduction, la patine résulte à la fois du «temps qui passe», en ce que ce temps-là affecte la surface des objets, en modifie les propriétés sensibles (forme, couleur, texture, pour l'essentiel), et du «temps qui dure», puisque la notion même de patine ne peut s'appliquer qu'à des objets solides, de forme stable et d'usage permanent.

Si on traite l'objet comme un corps (quitte à se poser plus tard la question de la manière dont les choses-corps deviennent des actants-objets), alors on peut lui appliquer la distinction banale entre la «substance» constitutive, la chair, et la frontière qui le sépare de tout ce qui n'est pas lui, l'enveloppe. Le «temps qui passe» affecte l'enveloppe; le "temps qui dure» concerne la chair matérielle même de l'objet.

Mais on s'aperçoit immédiatement que le propre de la patine est de modifier le rapport entre ces deux aspects de l'objet. En effet, avec le temps et avec l'usage, plus les objets sont patinés, plus ils se «ressemblent» superficiellement, de par les modifications plus ou moins uniformes de leurs enveloppes, tout en différant intérieurement, dans leur matière, leur structure, et leurs fonctions. Mais cette situation est destinée à évoluer encore, car, plus ils sont patinés, plus la couche affectée est importante, et plus la part de l'intérieur, supposé les différencier, diminue: à la limite, quand les objets sont au bord de l'usure définitive et au moment d'être abandonnés à la décharge, cette part de «chair» constitutive est devenue si difficile à identifier que l'usager a décidé de se séparer d'eux définitivement. En somme, plus ils ont quelque chose en commun, plus ils perdent de leur valeur d'usage.

Cette tension doit être plus précisément identifiée, puisqu'elle est la condition de la constitution d'une «interobjectivité». Tout d'abord en précisant le statut sémiotique des deux «parts» de l'objet.

La «chair» matérielle et fonctionnelle procure la définition formelle de l'usage: quel programme accomplir, avec quel rôle actantiel, grâce à quelle modalité dominante, et pour produire quel résultat; la structure de l'objet thématise en somme ces programmes et ces rôles. La forme et la structure du fauteuil le destinent à accueillir un acteur qui s'assoit, avec un rôle de sujet d'état, doté d'un pouvoir-faire, au bénéfice de quelque programme de base (se reposer, lire, etc.). Toute modification de la structure interne, usure lente ou détérioration brusque, remet ces rôles et ces programmes en cause, voire en suspend les conditions minimales. Comme l'enveloppe est en partie solidaire de la structure fonctionnelle, il ne s'agit pas seulement du tissu qui recouvre le fauteuil, mais de l'ensemble des parties de la structure qui peuvent être affectées par l'usage et l'environnement, et la patine doit respecter ces "conditions minimales" (thématiques, modales et narratives).

Mémoire figurative et surface d'inscription

La question qui nous occupe, celle des «sociétés d'objet» suscitées par la patine, implique que les figures aient en quelque sorte une certaine "mémoire", et que cette mémoire leur soit collective. Cette étrange implication mérite un détour.

Greimas insistait, notamment pour justifier la syntaxe du carré sémiotique ${ }^{2}$, sur la mémoire $d u$ discours: une affirmation qui suit une négation n'a pas la même valeur qu'une affirmation qui en suit une autre. La question de la mémoire du discours est pourtant beaucoup plus générale, et on peut même 
dire qu'elle concerne tout univers sémiotique, même le monde naturel, pourvu qu'on puisse y reconnaittre une syntaxe. Cette mémoire serait, comme dans les systèmes physiques quantiques, la mémoire des interactions entre entités sémiotiques: on admet bien, par exemple, que la rencontre entre un sujet et un antisujet est susceptible de les transformer durablement tous les deux, et d'augmenter le pouvoir faire, le savoir faire ou le vouloir faire du sujet; on admet aussi que la rencontre sensible et affective entre un sujet et un objet de valeur soit susceptible de transformer durablement ses passions. Mais il faudrait aussi admettre que les interactions entre figures sont aussi susceptibles de les modifier durablement, dans la perspective d'une syntaxe figurative.

Dans tout univers figuratif, et tout particulièrement dans le monde naturel, tout déploiement d'énergie, tout mouvement d'une seule figure est susceptible de modifier durablement l'enveloppe d'une ou plusieurs autres: c'est l'érosion éolienne des reliefs, c'est la trace de pas sur le sol, c'est l'objet renversé ou brisé par le passage d'un être animé. Cette suggestion ne doit pas surprendre: elle découle tout simplement du principe selon lequel toute la syntaxe figurative repose sur l'interaction entre matière et énergie, et que les équilibres stables ou instables de cette interaction produisent des figures identifiables. Dès lors que les objets sont traités comme des corps en interaction, et pas seulement comme des figures abstraites, et pris dans une syntaxe figurative, l'interaction entre matière et énergie se met en scène comme interaction entre mouvements des uns et enveloppes des autres.

Nous appellerons «marquage» ce principe syntaxique général de modification durable des entités sémiotiques par les interactions antérieures: ce principe suppose au moins que ces entités, outre leur rôle purement formel, obéissent à un autre principe, celui d'identité et de permanence. La chaîne ou la syntaxe des "marquages» constitue la «mémoire» du discours et, à ce titre, elle ne diffère pas des autres phénomènes syntaxiques évoqués plus haut (les modifications modales et passionnelles qui affectent les sujets dans leurs parcours). Enfin, dans le cas particulier des entités figuratives, et tout particulièrement des figures traitées comme des corps, alors les "marquages" sont des "empreintes", et la «mémoire» du discours, constituée dans ce cas particulier par le réseau de ces empreintes, formera ce que nous avons appelé jusqu'à présent la "surface d'inscription». La surface d'inscription est en somme la mémoire figurative d'un univers sémiotique, cette enveloppe constituée de la totalité des «souvenirs" de stimulations, interactions et tensions reçues par la figure-corps.

Cet ensemble conceptuel, marquage, mémoire, empreinte, mémoire figurative, surface d'inscription, est à mettre au compte d'une constitution de la syntaxe figurative du discours. L'empreinte peut être individuelle, mais la mémoire figurative est collective et ne saurait dissocier telle interaction de telle autre; en outre, la valeur de l'empreinte n'apparaît que par sa position dans ou sur la surface d'inscription tout entière. Le caractère collectif de cette étrange «mémoire» semble donc assuré.

\section{De la praxis énonciative à l'empreinte}

L'enveloppe de l'objet est ce qui en fait une «figure» reconnaissable, individualisable et perceptible. L'empreinte de la patine est inscrite sur cette enveloppe par les traces accumulées de l'usage: littéralement, donc, elle résulte du fait que des acteurs individuels et concrets ont assumé le programme et les rôles proposés par l'objet, qu'ils se les sont appropriés les uns après les autres, jour après jour. En ce sens, l'empreinte est le strict équivalent d'une trace énonciative, d'une inscription, dans l'énoncé même de l'objet, de la chaîne répétitive des actes dont il a été l'instrument, le lieu ou l'occasion.

Mais en tant qu'empreinte même, elle ne peut résulter que d'un très grand nombre de ces traces, qui, seul, peut expliquer une modification durable de l'enveloppe: dès lors, associée à une série indéterminée d'actes et d'usagers, la patine apparait comme une trace de la praxis énonciative ${ }^{3}$, et d'une énonciation collective et neutre quant à la personne ${ }^{4}$. Mieux que tout autre, elle est un parfait exemple d'un indice non subjectif de l'énonciation ${ }^{5}$. 
La tension dont nous faisions état est donc une tension entre la structure thématico-narrative de l'objet et l'indice d'une praxis énonciative collective; autrement dit, entre la structure (thématico-narrative et modale) et l'empreinte (énonciative, impersonnelle).

Comme nous le suggérions, l'empreinte se développe au détriment de la structure, ce qui suppose un autre aspect de la même tension: l'enveloppe de l'objet semble alors devoir concilier deux rôles: d'un côté, son rôle d'enveloppe proprement dite, qui signifie et manifeste la stabilité figurative d'une icône, et, de l'autre, le rôle que lui confère la patine, qui est d'être la surface d'inscription d'une empreinte (l'énonciation collective). La tension entre les deux dimensions de l'objet peut donc être comprise comme une tension entre deux rôles différents de l'enveloppe: contenir, maintenir, stabiliser d'un côté (l'icône); inscrire, imprimer, de l'autre (l'empreinte).

Ce que les objets partagent, dans l'«air de famille» que leur donne la patine, c'est bien entendu l'empreinte, et c'est donc le rôle de surface d'inscription qui est pertinent dans cette affaire. Néanmoins, l'autre rôle, celui de contenance et de maintenance, n'est pas pour autant indifférent: on ne s'aviserait pas, par exemple, de s'intéresser à la patine d'objets mis au rebut, d'objets cassés, définitivement usés ou méconnaissables. La valeur de la patine (de l'empreinte) dépend donc du maintien de la structure de l'objet (de l'icône); ou, en d'autres termes, la patine n'a de valeur, aussi importante et profonde soit-elle, que si la structure de l'objet est encore reconnaissable.

La surface d'inscription (portant l'empreinte d'un indice énonciatif) ne vaut donc qu'en raison de l'équilibre qu'elle entretient avec l'enveloppe de contenance et de maintenance (préservant la structure thématico-narrative). Certes, la patine est un signe de l'usure, mais d'une usure sans excès, car l'objet n'est objet (c'est-à-dire actant) que si toutes ses propriétés sont globalement conservées.

Pourtant, d'autres types de valorisation sont envisageables, mais ils supposent un déplacement du statut de l'objet de valeur: par exemple, le collectionneur se soucie moins de la conservation des fonctions de l'objet, pourvu que la structure et la matière en soient préservées; mais il accorde alors moins d'importance relative à son statut d'instrument, adjuvant ou prothèse. À la limite, pourquoi ne pas imaginer un collectionneur d'objets détruits ou incomplets: il ressemblerait fort à un archéologue, pour qui l'objet ne vaut plus que comme témoignage, document et source d'information sur le passé; mais, paradoxalement, c'est aussi ce type de collectionneur archéologue qui accorderait le moins d'importance à la "patine», ou même, le cas échéant, qui y verrait un obstacle à ses investigations. Décidément, il semble bien que la valeur de la patine ne puisse être conçue sans cette tension et cet équilibre avec la préservation de la structure matérielle, fonctionnelle et iconique.

Types énonciatifs et types de sociétés d'objets

La patine des objets implique, de fait, deux énonciations différentes: tout d'abord, celle qu'elle affiche en tant que patine actuelle et qui, reposant sur une inscription dans la matière de l'objet, proclame l'ancienneté de celui-ci et l'ensemble des contenus temporels, aspectuels et axiologiques associés à la figure de la patine; ensuite, celle dont nous faisions état plus haut, à savoir la série des "énonciations" individuelles qui, en actualisant jour après jour les fonctions et la structure de l'objet, le marquent peu à peu de leur empreinte. Globalement, l'ensemble de ces deux dimensions de l'énonciation forme ce que nous avons appelé la praxis, mais seule la première a le pouvoir de réinterpréter l'histoire de l'objet pour lui conférer les valeurs et les contenus attachés à la patine.

D'autres types d'énonciation, différents de celui de la patine, se dessinent alors, et notamment ceux qui, sous couvert de pratiques «maniaques» (celle du collectionneur) ou «scientifiques» (celle de l'archéologue), débouchent sur d'autres conceptions de l'«interobjectivité».

Pour l'archéologue, la coprésence des mêmes fragments ou objets sur un même site ne vaut que si elle atteste d'une coprésence antérieure d'usagers disparus, sur une période historique restreinte; en effet, un amas d'objets obtenu par accumulation sur 
une longue période historique n'indique rien de l'interaction entre eux: ces objets ne témoignent alors que de la récurrence de l'occupation des lieux, et ne permettent pas de reconstituer un «mode de vie»; il en va tout autrement si on peut prouver qu'ils ont été témoins, ensemble, de la vie quotidienne d'une même population. L'interobjectivité que l'archéologue appelle de ses vœux est par conséquent, grosso modo, synchronique, et elle est le gage d'un mode de vie homogène: un effet d'identité, en somme.

Cette identité se suffira de la représentativité des objets: pour qualifier un mode de vie, en effet, il faut et il suffit que les pratiques typiques d'une culture soient représentées: les pratiques alimentaires, rituelles, artistiques, etc. C'est pourquoi la patine et le nombre d'objets comptent peu dans ce cas: peu importent l'état de conservation et le nombre d'exemplaires, pourvu que les objets typiques soient présents et reconnaissables.

Pour le collectionneur, au contraire, la dispersion temporelle n'est pas un obstacle; au contraire, elle procure à l'ensemble des objets rassemblés une profondeur temporelle qui en fait tout le prix. Mais, du même coup, et paradoxalement, l'interaction entre les objets d'une même collection n'ajoute pas grand chose à leur valeur individuelle, puisqu' ils ne sont pas censés avoir cohabité dans le passé; à l'inverse, la relation individuelle entre l'objet et son ancien propriétaire devient prédominante: le sabre de Napoléon, la tabatière de Balzac, le fauteuil de Mitterand deviennent alors les pièces maîtresses d'une collection et, si leur patine intéresse, c'est seulement comme empreinte d'un usager d'exception, et non comme témoignage de leur improbable coexistence.

De fait, l'unité recherchée par le collectionneur, comme d'ailleurs par le conservateur de musée, est thématique: une isotopie suffit à faire une collection, pourvu que les objets rassemblés présentent tous au moins un trait figuratif qui témoigne de leur appartenance à la thématique retenue; de fait, le critère de l'interobjectivité est ici la généricité. Mais, par ailleurs, la valeur de la collection, et donc de chacun de ses éléments, repose sur le nombre: le collectionneur vise aussi à l'exhaustivité, et c'est même l'autre face de la passion qui l'habite: ne recueillir que les objets d'un certain genre, mais les posséder tous.

Revenons à la patine: en tant qu'énonciation, elle n'impose pas l'intervention d'un acteur extérieur, comme l'archéologue ou le collectionneur, tout au plus un observateur, qui en fait simplement le constat. Elle ne requiert ni thématique dominante, ni exhaustivité, ni représentativité, ni même une coexistence synchrone des objets et des usagers. Le moindre accessoire, futile ou sérieux, central ou marginal dans les pratiques, est transformé par la patine, car il porte la signature du temps et de ses usagers; et le rassemblement des objets patinés n'implique même pas qu'ils aient la même apparence superficielle: chacun est supposé en effet réagir à sa manière, selon la matière qui le compose, et les usages auxquels il a été soumis.

Pourtant, il faut bien qu'une unité se dégage, au moins un principe de "connivence» que manifeste la patine; ce principe est celui de la «ressemblance de famille», une distribution aléatoire et irrégulière de quelques traits sur le groupe d'objets rassemblés: quelques tâches ici ou là, quelques signes d'usure, quelques couleurs défraîchies, quelques marques de chocs ou empreintes sur les surfaces, chaque type de traces n'étant présent que sur un petit nombre d'objets. La patine constitue donc l'interobjectivité comme un réseau de traces, qui nappe l'ensemble des objets d'un semblant d'unité: nous dirons, pour faire bref, d'une familiarité. Le contenu de ce principe d'unité n'est ni thématique ni sociohistorique, mais tout simplement temporel et praxique. Ce réseau peut être lâche et dispersé, et les traces rares, mais il peut aussi être dense, abondant, et saturer l'ensemble des objets.

Représentativité, exhaustivité, familiarité: les énonciations dont nous recherchons les types se présentent d'abord comme des «principes d'unification", comme des modalités concrètes de regroupement, sur lesquelles repose leur mode de valorisation spécifique ${ }^{6}$. La représentativité est fondée sur le choix des «meilleurs exemplaires» dans chaque catégorie de pratique, et donc sur une visée sélective; 
la généricité repose sur un principe d'équivalence minimale entre les différents objets, le partage d'un trait commun systématiquement répété; l'exhaustivité, sur une visée quantitative et cumulative; la familiarité, enfin, sur une visée distributive et non cumulative.

Le principe général, permettant de définir plus précisément le type d'énonciation de la patine, par contraste avec d'autres, est celui d'une articulation de l'intensité et du nombre: intensité de l'affirmation d'identité ou de communauté entre les objets, et nombre des objets ou des marques qu'ils portent. La société des objets s'affirme alors soit par la quantité au détriment de l'intensité (généricité et exhaustivité de la collection), soit par l'intensité au détriment de la quantité (représentativité des objets découverts): dans ces deux premiers cas de figure, l'augmentation de l'intensité suppose une diminution de la quantité, et inversement.

Le cas de la patine est bien différent, puisque l'effet de l'identité augmente en proportion du nombre de traces et du nombre d'objets affectés par ces traces, et inversement. La patine conjugue l'intensité identitaire et le nombre, au lieu de les opposer; elle peut conjuguer leurs degrés faibles, tout autant que leurs degrés forts, assumant ainsi, selon le cas, une énonciation en sourdine ou éclatante. Peutêtre faudrait-il distinguer ici, et nous ferions alors écho à nos propos antérieurs, la patine et l'usure, la première pour l'énonciation «en sourdine», et la seconde pour l'énonciation éclatante.

Les principes d'unification entre les objets forment par conséquent un système, qui a la forme d'une structure tensive, réglée d'un côté par l'axe de l'identité (plus ou moins intense) et de l'autre par celui de la quantité?

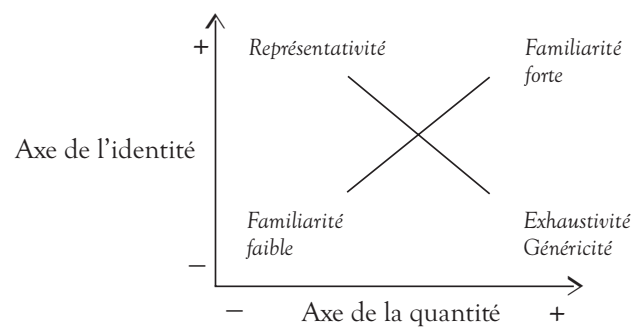

Les quatre types principaux ne sont que des positions extrêmes et laissent place à des positions intermédiaires, qui peuvent varier en continu. Les énonciations évoquées, celle du collectionneur comme celle de l'archéologue, ne sont que des cas particuliers: d'autres pratiques interobjectives pourraient occuper les mêmes positions. De même, la patine n'est qu'une des manières d'imposer un «air de famille» à un groupement d'objets; mais elle est la seule qui y parvienne par l'accumulation temporelle des usages et de la présence. Il faut donc la spécifier plus précisément, en revenant à cette condition particulière de son efficacité sémiotique.

\section{TRADITION ET CONTINUITÉ}

La patine n'énonce la communauté des objets que sous une triple condition: (1) la coexistence actuelle entre les objets conduit à l'hypothèse d'une coexistence antérieure; (2) la relation avec les éventuels usagers ne vaut que comme interaction globale, et l'hypothétique coexistence antérieure doit pouvoir être considérée comme le témoignage d'un mode de vie collectif; (3) la coexistence actuelle détient encore une signification pour elle-même, notamment comme «pérennisation» du mode de vie antérieur.

La "collection" ne satisfait ni à la première ni à la seconde conditions; si elle satisfait à la troisième, c'est uniquement dans le cas où la thématique du collectionneur s'y prête, et où son activité repose sur la nostalgie d'une époque antérieure: mais on sait bien, par exemple, que les nostalgiques du nazisme se satisfont bien souvent d'emblèmes et d'objets neufs, et que la patine leur importe peu.

Les objets découverts et/ou rassemblés par l'archéologue satisfont à la première et à la seconde conditions, mais pas à la troisième: ils ne font que témoigner du mode de vie antérieur, et ne figurent, en tant que rassemblement actuel, que comme «documents» synthétisant une information à découvrir; c'est pourquoi leur état de conservation ne vaut qu'en raison de leur valeur documentaire, et la patine n'est, au mieux, qu'indifférente. Ce cas de figure peut pourtant nous renseigner, par contraste, sur le rôle de la 
patine: l'archéologue travaille sur des époques oubliées, méconnues, et son activité n'a de sens que si on suppose de grandes ruptures temporelles, sociales et historiques, notamment dans l'usage des objets: on n'imagine pas en effet un archéologue s'intéressant à des ensembles d'objets qui, même anciens, n'auraient jamais cessé d'être là et d'être utilisés; inversement, c'est justement l'absence de ruptures temporelles qui donne toute sa valeur à la patine.

Une nouvelle condition de la patine apparaît, qui n'est rien d'autre que la reformulation de la troisième condition précédente: une condition de continuité, dont dépend la continuité d'une coprésence des objets, et la permanence d'une «forme de vie». Reste à comprendre dans quel système axiologique s'inscrit cette condition.

\section{La chose, l'objet et l'empreinte}

Nous évoquions tout à l'heure le temps: au moment où l'observateur constate l'existence d'une patine, ce temps écoulé se trouve à la fois suspendu (la question ne se pose plus de savoir si la patine va augmenter), convoqué dans la matière, et signifié par l'empreinte. On pourrait être tenté alors de parler d'une "présentification de l'absence»: mais cette expression, si elle convient bien dans l'analyse que Greimas proposait de la nostalgie, est ici déplacée.

Tout d'abord, ce que convoque la nostalgie, c'est seulement un simulacre, un simulacre efficient, certes, puisqu'il affecte le sujet; il est clair que la nostalgie et le plaisir qu'elle procure se nourrissent de l'absence de la chose convoquée, alors que la valeur de la patine repose entièrement sur la présence tangible de l'empreinte. Cette étrange présence du passé doit être élucidée.

Ensuite, même si l'attrait pour la patine peut prendre sa source dans une complaisance nostalgique, l'effet de sens de la patine elle-même n'a rien de nostalgique, ni même de passéiste: ce qui importe ici encore, c'est la trace présente de l'usage, et le sentiment qu'elle procure de pouvoir se couler dans les schèmes établis et les valeurs d'une «tradition», en reproduisant cet usage.
Examinons pour commencer cette «étrange présence» du passé dans la matière. Ce qui se donne à appréhender est une chose et l'empreinte de la patine en fait un objet; imaginons que nous découvrions une chose inconnue: la seule présence d'une "patine" peut nous indiquer, selon la nature de cette patine, qu'elle a été fréquentée et utilisée par des êtres animés, et que, par conséquent, elle a pu jouer un rôle et figurer comme instrument dans des pratiques oubliées; c'est ainsi que raisonnent, par exemple, les archéologues qui découvrent des silex taillés. Nous n'avons alors plus accès à la structure de l'objet, mais seulement au témoignage de ses usages; ce n'est qu'ensuite que nous pouvons nous interroger sur la structure de l'objet.

Cette relation entre la chose et l'objet ne peut être établie que grâce à une mémoire inscrite dans la figure de l'empreinte.

Par définition, dans l'empreinte, rien ne sépare la chose de l'objet, sinon un changement de statut et, surtout, une disjonction spatio-temporelle: quand il s'agit d'un masque funéraire, par exemple, le moule conserve une forme dont la chair a disparu, mais que n'importe quelle autre matière peut venir remplacer; quand il s'agit de l'empreinte relevée par l'enquêteur, la figure établit le lien entre une partie du corps de celui qui, d'une part, est passé sur les lieux, et qui est supposé avoir accompli un forfait, et, d'autre part, une partie du corps d'un $\mathrm{X}$ à identifier, qui habite quelque part, qui a des habitudes, un métier, une famille, etc. Dans le premier cas, il s'agit du même visage, l'un étant de chair, l'autre fait d'une autre matière; dans le second cas, il s'agit du même pied ou de la même main, mais avec un changement de rôle figuratif.

L'empreinte réalise les deux conditions d'une «présence» des usages passés: (1) une contiguité parfaite, sans hiatus, qui assure la continuité d'une présence, et (2) un nécessaire basculement des modes d'existence, qui explique le changement de statut de cette même présence. La première condition est si bien réalisée que l'empreinte est considérée comme témoignage, preuve, signature individuelle: à cet égard, elle assure une parfaite continuité entre la chose et ses usages d'objet; la seconde l'est tout 
autant, car pour que l'empreinte puisse fonctionner en tant que telle, il faut que la chose ne soit plus perçue comme telle, et que l'objet s'impose: aussi longtemps que la main reste posée sur la surface où elle appose ses traces, il n'y a pas, à strictement parler, d'empreinte; aussi longtemps que le visage moulé reste dans le moule, il n'y a pas de moulage, mais un simple masque de plâtre ou d'argile. Il faut que l'objet et la chose soient l'un actuel et l'autre potentielle pour que l'empreinte puisse fonctionner comme «signe» et impliquer ainsi des processus interprétatifs et persuasifs, des stratégies de réminiscence et de témoignage, etc. Il faut en somme un «débrayage» de la surface où s'inscrit la patine.

Mais cette analyse de l'empreinte, comme garantie de la présence, ne vaut pas seulement dans le cas de la patine, puisqu'elle pourrait tout aussi bien convenir au travail de l'archéologue.

\section{Énonciation et tradition}

Maintenant, la tradition et ses valeurs. C'est un motif bien connu du fonctionnement de la tradition que celui de la continuité temporelle et spatiale de sa transmission. Le moindre hiatus dans la chaîne de la transmission compromet la croyance et affaiblit la tradition. Maintenir une tradition, c'est donc avant tout en saturer les relais énonciatifs: la tradition n'est vivante que si on peut reconstituer ou imaginer une chaîne temporelle ininterrompue d'énonciations, car cette continuité sans faille garantit la présence maintenue et potentielle de l'origine.

En somme, la tradition est une propriété de la praxis énonciative (la saturation des relais énonciatifs) qui donne un accès direct, immédiat et crédible aux valeurs de l'origine. Mais il s'agit d'une énonciation impersonnelle qui, à chaque relais, est réactivée sous forme personnelle. Certes, on ne peut se passer d'un "impersonnel», d'une instance collective et débrayée qui garantit la fiabilité de la chaîne. Mais il faut aussi, pour que la tradition fonctionne, que des énonciations personnelles viennent à chaque moment vivifier cet impersonnel; la tradition n'est efficiente dans le présent, justement, que parce que des énonciations personnelles peuvent l'actualiser et s'en réclamer, et parce que ces énonciations personnelles restent en tension avec l'impersonnel sans se fondre en lui.

La patine est une figure sensible qui réalise point par point chacune de ces propriétés: certes, elle ne garantit pas une absolue continuité de l'usage, mais elle est mieux qu'une figure de la continuité des usages: par superposition, compacification et accumulation, elle en réalise la présentation simultanée. Elle assure aussi la présence de l'origine, puisque la chaine des usages inscrite sous forme d'empreinte assure au moins le sentiment d'une conformité aux programmes, rôles et propriétés inscrits dès le départ dans la structure de l'objet. Elle implique aussi une dialectique de l'impersonnel et du personnel, en invitant chacun à prendre place individuellement et personnellement dans la chaîne des usages: en cela, en tant que figure de la tradition, la patine articule soigneusement la rétention - l'énonciation collective et impersonnelle de l'empreinte - et la protension - l'énonciation personnelle et individuelle de chaque nouvel usage; il est clair que cette articulation nécessaire la distingue de l'approche archéologique des objets anciens.

Empreinte, tradition et société des objets

En quoi tout cela témoigne-t-il de la cohabitation des objets?

La figure de l'empreinte ne prend tout son sens que si elle permet de reconstituer l'ensemble d'un parcours, d'une activité ou d'une situation: un enquêteur qui, voyant une trace de pas, se contenterait de conclure que "quelqu'un est passé par là", n'aurait pas beaucoup d'avenir; de même, un observateur qui, contemplant un objet patiné, se contenterait d'en conclure qu'il a beaucoup servi, semblerait peu perspicace. Le fait est que l'empreinte est une trace qui, tout en ne renvoyant, en tant qu'indice énonciatif, qu'à un segment étroit de l'usage, suscite tout un programme interprétatif: dès lors, une empreinte ne vaut que si elle est confirmée par d'autres indices, si elle corrobore des hypothèses, si elle permet de construire des inférences créatives. À cet égard, l'empreinte, tout comme un lexème peut être le 
condensé d'un récit, est le condensé d'une configuration beaucoup plus complexe.

La patine d'un objet déclenche un processus interprétatif qui ne s'alimente et ne se satisfait que de la patine des objets voisins: ainsi, de trace en trace, un mode de vie renaît peu à peu. La "communauté» des objets patinés fonctionne alors comme le réseau des indices pour l'enquêteur conformément au principe de familiarité évoqué plus haut, et la patine des uns appelle, pour confirmation ou complément d'information, la patine des autres; dès lors, ce n'est pas seulement dans la chaîne des usages d'un objet que se glisse l'observateur, mais dans une véritable «forme de vie» ${ }^{8}$, dont il scrute et met en relation des traces sensibles.

D'un autre côté, croire en une tradition, c'est postuler l'unité fondamentale et peut-être inapparente entre des objets, des pratiques et des discours apparemment hétérogènes: de même, la patine, comme témoignage de la tradition d'un usage, invite à postuler l'unité profonde de la forme de vie qui peut se lire dans le rassemblement d'objets hétérogènes.

Revenons un bref instant aux autres formes d'«interobjectivité». Chez le collectionneur, l'interobjectivité n'est lisible que dans la thématique de la collection, et elle n'est assurée que par l'investissement affectif que le collectionneur luimême (et non les usagers) place dans cette thématique, plus que dans les objets eux-mêmes; cette dimension compulsive fait du collectionneur, typiquement, ce que J. Geninasca appelle un «sujet voulu " 9 . On aurait alors affaire à une interobjectivité régie par la modalité vouloir. Chez l'archéologue, nous l'avons assez souligné, l'objet ayant le statut de «document» et ne valant que par le poids d'information qu'il est susceptible de délivrer, l'interobjectivité n'est régie que par la modalité savoir. En revanche, pour ce qui concerne la patine, le rôle de la tradition, l'importance de la continuité des relais énonciatifs, l'adhésion intime au système de valeurs sous-jacent, qu'implique la reproduction actuelle à l'identique des usages anciens, tout cela confirme que le rassemblement des objets opéré par leur patine commune relève de la croyance: l'interobjectivité serait alors régie par la modalité du croire.

\section{LA PATINE ET L'ERGONOMIE.}

Interfaces

Dans l'analyse sémiotique des objets ${ }^{10}$, on distingue deux types d'interfaces: l'interface sujet et l'interface objet. L'interface sujet (ou «interface usager») est celle qui, par exemple dans un outil, facilite la "prise» manuelle, ou, dans un logiciel, facilite l'accomplissement d'une tâche. L'interface «objet» est cette partie de l'outil qui est formée, adaptée, optimisée pour entrer en contact avec les choses du monde, pour y tailler, gratter, enfoncer quelque chose; c'est aussi l'interface "objet» qui, dans un logiciel, permet de transformer les actions de l'usager sur le clavier ou sur l'écran en opérations portant sur le système de l'ordinateur et sur les données qu'il contient.

Lorsque l'archéologue préhistorien reconnaît un «instrument de percussion», il faut qu'il ait pu identifier à la fois l'interface objet qui lui indique le type de transformation que l'objet impose à la matière des choses et l'interface sujet qui lui indique quel geste est nécessaire pour accomplir la transformation. Ces deux interfaces sont aussi celles mêmes qui concentrent toute l'attention des ergonomes.

Mais la patine ne procède pas autrement; il suffit d'examiner un marteau d'ardoiser pour s'en convaincre: le manche est creusé à la longue par l'emplacement des doigts, la tête métallique est arrondie en son extrémité de percussion et entaillée sur sa longueur par le frottement répété de la tranche des ardoises, après cassure. L'outil a pris, plus que la forme de l'objet ou de la main, la forme même des gestes qu'il a servi à accomplir.

Modalités et types de sociétés: communication ou connivence

L'interface est le produit du premier type d'énonciation: celui qui, anonyme ou bien identifié, personnel ou impersonnel, inscrit l'empreinte sur l'objet. Mais, du point de vue de l'autre énonciation, celle qui s'approprie l'objet et en réactualise l'usage, 
l'effet est essentiellement modal, et la structure modale de la patine n'est pas la même que celle de l'ergonomie.

Dans le cas de l'ergonomie, à partir d'une représentation schématique et idéale du geste à accomplir, un actant distinct de l'usager, une sorte de destinateur transcendant, inscrit dans la matière de l'objet un "pouvoir faire» et un «savoir faire» optimisés et destinés à l'usager. Mais, pour l'usager, cette forme constitue une contrainte et devient donc un «devoir faire»: il suffit d'essayer de placer les doigts, dans les anneaux «ergonomiques» d'une paire de ciseaux, d'une autre manière que celle prévue par le constructeur, ou même d'être gaucher, pour s'en convaincre immédiatement. On aurait donc affaire dans ce cas à une structure énonciative très classique, une relation de communication entre un énonciateur et un énonciataire, et une relation prescriptive, qui ferait de l'énonciataire un usager soumis à la représentation que l'énonciateur s'est fait de lui au moment de concevoir l'objet.

En revanche, dans le cas de la patine, la déformation imposée à la surface de l'objet ne résulte que de l'exercice d'un "pouvoir faire» et d'un «savoir faire", et de l'adaptation progressive de la surface de l'objet à cette action répétée: en somme, on a toujours affaire à une inscription matérielle du "pouvoir faire» et du «savoir faire», mais dont l'initiative revient à l'usager, sous la forme d'une appropriation et d'une spécification individuelles de l'objet. En ce sens, le geste qui réactualise l'usage de l'objet ne diffère pas de ceux qui ont inscrit l'empreinte: il en prolonge la série, il se coule dans la même position énonciative, il participe de l'inscription elle-même. La relation énonciative n'est plus une relation de communication, mais de "communion" ou de "connivence», et repose sur une simple identification d'une énonciation à d'autres énonciations.

Dans un cas, l'empreinte a valeur transcendante, générique et contraignante; dans l'autre, elle a valeur immanente, spécifique, et auto-adaptative.

L'une et l'autre constituent ce qu'on pourrait appeler des «sociétés d'objets», mais, justement, des sociétés réglées par des systèmes actantiels, modaux et axiologiques fort différents.

Une famille d'objets ergonomiques se rassemble (et se ressemble) au nom de la rationalisation du geste, de l'optimisation de l'action et d'une schématisation transcendante, générique et contraignante de la vie quotidienne; le paradoxe de l'ergonomie, c'est qu'en adaptant les objets à leurs usagers, elle standardise l'usage et uniformise la forme des objets: cela implique, bien entendu, que l'usager auquel elle prétend s'adapter est un artefact idéalisé, un genre presque universel; dans ce cas, de fait, c'est l'activité en tant que schéma générique que l'on modélise, et non l'usager.

Une famille d'objets patinés, en revanche, se rassemble au nom de l'appropriation et de la spécification individuelles des objets par leurs usagers, même si, au bout du compte, et le temps passant, l'effet global est, comme nous le soulignions plus haut, collectif et impersonnel. La patine, en effet, même si elle relève d'une énonciation impersonnelle, fonctionne comme une signature, la signature d'un collectif indéterminé, mais libre d'imprimer sa marque dans son environnement. L'activité s'y trouve là aussi schématisée, mais par accumulation et renforcement progressif des modifications qui apparaissent aux points d'impact ou de contact, et non par le choix d'un quelconque "genre" abstrait.

Dans un cas, l'empreinte procède d'une décision d'équivalence et d'un principe d'analogie: entre tous les gestes possibles, dans les limites d'un usage donné, il y a une ressemblance suffisante pour qu'on puisse en définir le type idéal. Dans l'autre cas, seules la contiguité et la répétition sont requises: entre tous les gestes possibles, pour un même usage, il y a des points de contact et d'impact proches, qui se superposent approximativement et qui dessinent ainsi l'empreinte des usagers.

Il y aurait donc des sociétés d'objets totalitaires et stéréotypées, dont l'organisation tout entière reposerait sur l'équivalence postulée entre les usagers, entre les usages et entre les objets afférents, et d'autres, démocratiques et créatives, qui 
découvriraient, par approximation et libre initiative individuelle, les chemins de la familiarité, de la connivence et de la vie en communauté.

\section{LA PATINE SANS L'USAGE:}

\section{LA CONSTITUTION DE L'ACTANT}

Nous avons jusqu'à présent confondu (volontairement) la patine et les effets de l'usage, voire, à la limite, les effets de la fréquentation des objets par des êtres animés. Et nous avons profité de cette confusion pour en décliner les propriétés d'empreinte, de signature, d'inscription d'une tradition... C'était en somme une manière de nous accorder une facilité, celle que procure la référence à un usage et à un usager: puisque, en bonne méthode, il n'y a pas d'objet sans sujet et réciproquement, il est plus facile de fonder l'interobjectivité sur la présence, même implicite, des sujets que sur leur absence.

Renonçons, pour finir, à cette facilité. Nous y sommes, de fait, incité par la définition même du dictionnaire, qui n'évoque rien d'autre, dans l'acception originelle, que l'effet du temps sur la surface des objets en cuivre.

La gare de ma ville natale, Limoges, dite «gare des Bénédictins", est entièrement couverte de cuivre: le dôme central aussi bien que le toit de la tour à l'horloge latérale. Ce toit en cuivre était oxydé, grisvert, comme il convient à un témoignage du passé. C'est un monument classé, datant des débuts du chemin de fer en France et de l'époque où le réseau national était encore partagé entre quelques grandes compagnies privées interrégionales; à l'époque, elle était au centre d'un important nœud ferroviaire et, à l'intérieur du dôme central, aux quatre coins de la voûte, quatre statues représentent encore les quatre régions qu'elle reliait entre elles. Récemment, ce dôme a été détruit par un incendie et le cuivre a dû être changé, mais en cuivre déjà «patiné». En revanche, la restauration de toute autre partie, et notamment des murs de pierre, vise, au contraire, à retrouver la couleur et la texture originelle du matériau. Le cuivre des toitures, en somme, ne vaudrait que par la patine particulière qu'il procure au monument.
Il est bien clair que, dans ce cas, la patine ne doit rien à l'usage: et même, dans ce genre de monument, on s'efforce, par l'entretien, par des restaurations et des aménagements périodiques, d'effacer les traces de l'usage, voire de moderniser les fonctions intérieures. La valeur de la patine ne doit donc rien à l'inscription des «énonciations» et des "appropriations " individuelles et collectives qui constituent l'histoire quotidienne du bâtiment.

Tout au contraire, c'est à une autre histoire qu'on se réfère ici: une histoire collective, certes, mais celle des institutions, des structures politiques, de la ville et du chemin de fer. La patine n'est plus une empreinte (ce n'est que par une métaphore approximative et animiste qu'on parlerait d' «empreinte du temps»), mais un emblème, un signe d'identité acquise par permanence et conservation, et plus précisément par la permanence d'une présence, en ce lieu qu'elle désigne de loin. L'empreinte est une figure inscrite dans la matière, et l'emblème, une figure adjointe et associée à l'objet. La patine des toitures indique même qu'elles n’ont jamais été touchées ni modifiées et, sous la contrainte d'une réparation après incendie, on fait même usage d'un simulacre, la patine artificielle, pour «faire comme si» cette permanence n'avait jamais été interrompue; mais on voit bien alors que l'adjonction de la figure n'en modifie pas l'efficacité : l'artefact est toujours un emblème. Ainsi, ces toitures peuvent-elles continuer à dialoguer à distance, comme elles l'ont toujours fait, avec les autres monuments emblématiques de la ville.

Dans ce cas, la «société des objets» repose non sur une actantialisation par les usagers, mais par l'histoire, considérée comme un actant immanent au déploiement temporel. L'actant d'énonciation qui se propose sous cette forme a le même statut que celui qui résulte de l'usage: faisant office de destinateur garant des valeurs, il est susceptible de les énoncer «au nom» d'une collectivité; mais à la différence de son proche parent, qui est collectif, et immanent à la continuité de l'usage, et qui suppose des réactualisations individuelles permanentes, celui-ci n'a rien de collectif: ce serait en quelque sorte l'autre 
version de la tradition, celle qui assume elle-même ses énonciations, celle qui, bien installée et pérenne, ne doit plus grand chose aux initiatives individuelles. Et même, si nous supposions plus haut que cet actant patiné peut "dialoguer" avec les autres monuments de la ville, c'est bien que nous lui reconnaissons un statut personnel: ainsi serions-nous passé du «ILS» au «ON». En effet, dès que la patine est considérée indépendamment des éventuels usagers, elle confère à l'objet le statut d'actant quasi personnel, ou plus précisément d'actant personnel neutre.

Il reste pourtant à comprendre comment se construit ce statut actantiel. Pour commencer, le monument en tant que signe architectural et urbain n'est plus un objet: c'est un actant sujet, certes faiblement modalisé, mais qui comprend au moins un «pouvoir faire» et un «savoir faire» qui lui sont propres, et grâce auxquels il peut communiquer l'identité dont il est l'emblème. On pourrait même considérer que la patine (ainsi que nous l'avons définie plus haut, comme manifestation d'une permanence, d'une conservation et d'une relative insensibilité à l'usage) sous-tend un faire persuasif: elle convainc en effet l'observateur de cette continuité et de cette immuabilité de la présence, qui en font tout le prix. Mais, avant d'en arriver là, il faut se demander comment, avant d'être sujet, le monument est, tout simplement, actant.

La concentration de l'effet emblématique sur la toiture en cuivre patiné oriente la réponse: le cuivre est ici, pour des raisons techniques, une simple feuille, mais, pour le profane ou le naif, rien ne permet de savoir qu'il s'agit d'une surface et non d'un volume massif; en revanche, la patine qui recouvre cette matière ne peut être qu'une surface, une enveloppe. Et même, pour en revenir à quelques considérations techniques, on sait qu'il s'agit d'une surface protectrice, car, une fois formée sous l'action de l'air, cette pellicule empêche la corrosion d'attaquer plus profondément la matière qu'elle recouvre. Dès lors, le monument est formé comme un corps: d'un côté, une structure, des matières et des fonctions, et, de l'autre, une enveloppe, par laquelle il communique avec l'environnement; l'environnement modifie la pellicule de surface, et cette modification protège la structure intérieure contre la poursuite de l'agression extérieure.

Ce dispositif est l'analogue approximatif de l'enveloppe corporelle, aussi bien du point de vue physique que psychique: réception des stimulis - excitations et agressions venues de l'extérieur-, adaptation et modification de la surface, et protection. D'un point de vue phénoménologique, on pourrait dire en ce cas que le bâtiment est l'enjeu d'une saisie analogique: nous reconnaissons en ce corps recouvert d'une enveloppe l'équivalent de notre corps, recouvert d'une peau qui communique avec l'extérieur. Voilà donc le monument transformé en actant par équivalence avec notre propre corps. Voilà donc aussi pourquoi cette enveloppe concentre tout l'effet persuasif et emblématique du monument: c'est elle qui communique et signifie l'identité préservée, en harmonie avec la ville.

\section{POUR FINIR}

La patine des objets aurait donc quelque chose à voir avec notre enveloppe polysensorielle, enveloppe projetée à partir de l'instance du Moi et qui offre aux figures du monde une surface d'inscription sensible à l'action du monde extérieur. Nous avons raisonné jusqu'à présent en partant de la constitution matérielle de cette enveloppe. Mais, si on se réfère à la constitution sémiotique de l'enveloppe corporelle, on doit se rappeler que l'opération d' "enveloppement" est, dans cette perspective, une propriété de la polysensorialité elle-même: l'ensemble des stimulations et des sollicitations intérieures et extérieures forme un réseau solidaire et relativement stable, qui constitue l'«enveloppe» du corps sensible, tout comme l'ensemble cumulé des stimulations et sollicitations de l'environnement forme à la longue un réseau d'impacts et de modifications, puis une «nappe» continue et une "enveloppe» des objets, qui se substituent peu à peu à leur enveloppe matérielle et fonctionnelle.

Cette propriété commune aux corps sensibles et aux objets patinés, à savoir l'enveloppe conçue comme un 
réseau de connexions, à la fois spatiales et temporelles, est aussi ce qui, quelles que soient leur hétérogénéité et leur incompatibilité de départ, les rend compatibles, voire harmonieux: en effet, même s'ils ne partagent aucune structure, matière ou fonction, même si la patine a sur eux des effets différents, ils partagent au moins ce réseau de sollicitations et de stimulations, qui les a progressivement enveloppés d'une même histoire, d'une même aura sémiotique.

\section{N O TES}

1. À l'occasion de ce numéro conçu et dirigé par E. Landowski et G. Marrone, la question de l'interobjectivité me donne l'occasion d'aborder indirectement, du même coup, un autre thème mis à l'honneur par E. Landowski, «l'Usure», au cours d'un colloque organisé à Urbino, avec G. Ceriani, et auquel je n'avais pu participer, malgré mon intérêt pour le sujet proposé.

2. A. J. Greimas et J. Courtés, 1979, entrée "Carré sémiotique».

3. Sur cette notion, on pourra consulter D. Bertrand, 1993, p. 25-32, J. Fontanille et C.Zilberberg, 1998, chap. «Praxis»; J. Fontanille, 1999b, chap. «Énonciation".

4. L'idée qu'une énonciation pourrait être «impersonnelle», avancée à la fois par D. Bertrand (1993) et par C. Metz (1991), mérite encore discussion. Dans le cas de la patine, par exemple, l'empreinte actuelle, bien qu'elle résulte d'une collection indéterminée d'énonciations individuelles, peut néanmoins être comprise comme un embrayage de cette collection d'énonciations révolues, embrayage sur l'actualité de l'objet. Dès lors qu'un embrayage est reconnu, la sphère de la personne est convoquée. Nous reviendrons sur cette question.

5. Dans le cas de la patine, l'énonciation en question connait une extension sujette à caution: en effet, les traces du temps ne sont pas seulement celles laissées par les usagers, et le simple fait, pour un objet, d'être là, quelque part, soumis aux conditions d'environnement de ce "quelque part", participe de la patine. Pourtant, quand la patine se donne à appréhender, les frontières entre ce qui relève d'une actualisation des fonctions par l'usage, d'une part, et ce qui découle de la participation à un environnement, d'autre part, s'effacent et laissent place à un effet global de praxis énonciative impersonnelle. Nous examinerons plus loin le cas de la patine «sans usage».

6. Sur le même principe, on trouvera dans J. Fontanille, 1999a, chap. "Point de vue", une présentation plus détaillée de ces "principes d'unification" et de leurs corrélats perceptifs et axiologiques. Sur la question théorique de la structure méréologique des objets, il faut se reporter à l'étude désormais classique de J.-F. Bordron, 1991.

7. Sur la définition et le fonctionnement de la structure tensive, on peut consulter J. Fontanille et C. Zilberberg, 1998, chap. «Valence» et chapitre "Valeur» ; et J. Fontanille, 1999b, chap. «Structures élémentaires".

8. Ce concept proposé par Greimas a été défini et illustré dans «Les formes de vie» (J. Fontanille, 1993); Tension et signification (J. Fontanille et C. Zilberberg, 1998, chap. "Forme de vie»). Une forme de vie est une déformation cohérente qui affecte l'ensemble des niveaux du parcours génératif d'un discours ou d'un univers sémiotique quelconque, depuis les schèmes sensoriels et perceptifs jusqu'aux structures narratives, modales et axiologiques. Dans le cas qui nous occupe, le «mode de vie" ancien est transformé en «forme de vie» dès lors qu'il est devenu sensible à travers les effets de la patine, qu'il est pris en charge globalement par une croyance qui atteste de sa cohérence, et qu'il est soumis au système de valeur particulier d'une tradition.

9. Cf. J. Geninasca, 1997, p. 31-32.

10. A Zinna, «Les objets et leurs interfaces», à paraître.

\section{RÉFÉREN CES BIBLIO G RAPHIQ U ES}

BertRAND, D. [1993] : «L'impersonnel de l'énonciation. Praxis énonciative: conversion, convocation, usage», Protée, vol. 21, no 1, 25-32. BORDRON, J.-F. [1991] : «Les objets en parties », Langages, no 103, p. 51 65.

FONTANILLE, J. (dir.) [1993] : «Les formes de vie», RSSI, vol. 13, nos 1-2; [1999a]: Sémiotique et littérature, Paris, P.U.F.;

[1999b]: Sémiotique du discours, Limoges, Pulim.

Fontanille, J. et C. Zilberberg [1998]: Tension et Signification, Liège, Mardaga.

GeninasCA, J. [1997]: La Parole littéraire, Paris, P.U.F.

Greimas, A. J. et J. COURTÉs [1979]: Sémiotique. Dictionnaire raisonné de la théorie du langage, Paris, Hachette.

METZ, C. [1991] : L'Énonciation impersonnelle ou Le Site du film, Paris, Méridiens Klincksieck.

ZINNA, A. "Les objets et leurs interfaces", dans J. Fontanille et A. Zinna (dir.), Les Objets au quotidien, Limoges, Pulim (à paraître). 